\title{
Gravity-Independent Oscillate Boiling
}

\section{Journal Article}

\section{Author(s):}

Nguyen, Dang Minh; Supponen, Outi (D); Miao, Jianmin; Farhat, Mohamed; Ohl, Claus-Dieter

Publication date:

2019-12

Permanent link:

https://doi.org/10.3929/ethz-b-000402315

\section{Rights / license:}

In Copyright - Non-Commercial Use Permitted

\section{Originally published in:}

Microgravity science and technology 31(6), https://doi.org/10.1007/s12217-019-09708-8 


\title{
Gravity-independent oscillate boiling
}

\author{
Dang Minh Nguyen • Outi Supponen • Jianmin Miao • Mohamed \\ Farhat • Claus-Dieter Ohl
}

Received: date / Accepted: date

\begin{abstract}
Heat transfer in nucleate boiling relies on the detachment and rise of the boiling bubble, in which gravity plays the dominant role. Previous studies showed that in the absence of gravity, the bubble fails to rise, causing the dryout of the heater and significantly reducing the thermal efficiency of nucleate boiling. Recently, a new boiling regime termed oscillate boiling was discovered. By localizing thermal energy as high as $50 \mathrm{~mW}$ into an area as small as $15 \times 15 \mu \mathrm{m}^{2}$, a boiling bubble is formed and oscillate at high frequency while remaining pinned at the heating spot. This regime was proposed to be independent of buoyancy as its operation does not involves the detachment and rise of the boiling bubble. To test this hypothesis, we compared experimental observations of oscillate boiling in low gravity, normal
\end{abstract}

Dang Minh Nguyen

School of Physical and Mathematical Sciences, Division of Physics and Applied Physics, Nanyang Technological University, Singapore 637371

Tel.: +65 84267026

E-mail: nguyenda001@e.ntu.edu.sg

Outi Supponen

Department of Mechanical Engineering, University of Colorado, Boulder, CO 80309, USA

E-mail: Outi.Supponen@Colorado.edu

Jianmin Miao

School of Mechanical and Aerospace Engineering, Nanyang Technological University, Singapore 639798

E-mail: Mjmmiao@ntu.edu.sg

Mohamed Farhat

Laboratory for Hydraulic Machines, Ecole Polytechnique Federale de Lausanne, CH 1007 Lausanne, Switzerland

E-mail: Mohamed.Farhat@epfl.ch

Claus-Dieter Ohl

Otto-von-Guericke University, Institute for Physics, Magdeburg 39106, Germany

E-mail: claus-dieter.ohl@ovgu.de gravity and hyper gravity. The results support the hypothesis and promote its potential for outer-space heat transfer application.

Keywords Microgravity · Boiling · Bubble · Oscillation · Thermal Management

\section{Introduction}

The development of space technology involves lightweight, compact and high-power devices, while at the same time requires strict temperature control to maintain proper operation. In ground applications, i.e. terrestrial condition, such tasks are managed by boiling heat transfer as it ensures high thermal efficiency and low temperatures of the dissipating devices [1]. Here, the heat transfer mechanism is attributed to the detachment and rise of the nucleate boiling bubbles [2], in which gravity plays an important role. However, in space with greatly reduced gravity, buoyancy-based boiling encounters serious limits. Earliest observation of pool boiling in microgravity reported by Abe et al. [3], Ohta et al. [4] using sounding rockets, and Oka et al. [5] on parabolic flights show that without buoyancy, boiling bubbles fail to rise. Instead, they grow, coalesce with their neighboring bubbles and hover close to the substrate's proximity. Later on, using a set of multiple temperature-controlled heating elements, Kim et al. [6] managed to capture the boiling curve and the substrate's temperature during the boiling process. The results reveal that as the bubbles fail to rise, they cause the dryout of the substrate and trap thermal energy within. Consequently, the heater experiences a significant rise in temperature together with a drop in the critical heat flux (CHF). The same observations have been reported in numerous studies on pool boiling with 
a

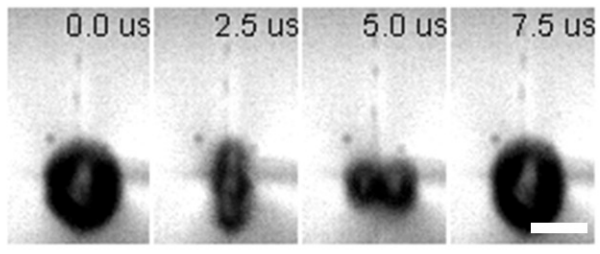

b

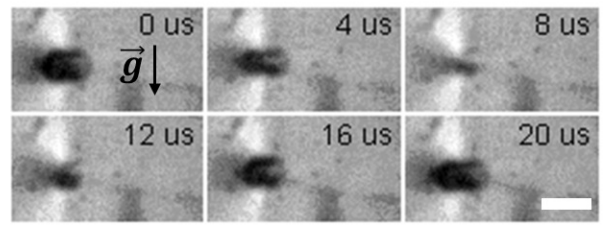

Fig. 1 (a) One typical oscillate boiling cycle. Here the heating power is $86 \mathrm{~mW}$. (b) Oscillate boiling preserves its characteristics when the substrate is oriented parallel to gravity. Here the heating power is $62 \mathrm{~mW}$. The scale bars in each figures represents $20 \mu \mathrm{m}$. different heater's geometries, test liquids [7-12], and microgravity platforms [13-16]. These works are extensively reviewed by Mudawar et al. [17] and Colin et al. [18]. Thermocapillary stress has been speculated to be capable of transporting heat from the substrate into the bulk liquid in the absence of gravity. However, its efficiency is still far inferior compared to convection in terrestrial condition [6,19-21]. Thus, utilizing pool boiling for outer-space thermal management remains a challenge.

Recently, we reported a new boiling regime termed oscillate boiling, which may provide a solution for micro gravity environments $[22,23]$. Unlike nucleate boiling, oscillate boiling is expected to be independent of gravity since its heat transfer is not dependent on the detachment and rise of vapor bubbles from the heater surface. Instead, by concentrating thermal energy between $50-100 \mathrm{~m} \mathrm{~mW}$ into an area as small as $15 \times 15 \mu \mathrm{m}^{2}$, a boiling bubble is formed that remains pinned on the surface while undergoing repetitive high frequency volume oscillation, up to several $100 \mathrm{kHz}$. Figure 1a shows captured images of one typical period of the oscillate boiling bubble. The presence of a solid boundary induces non-spherical collapses of this bubble [24]. As a result, a liquid jet is developed during each collapse and impacts onto the high temperature heater. Upon this impact, the jet is vaporized, resulting thermal energy being removed from the substrate and transported into the bulk liquid. The heat is carried by a flow driven by the thermocapillary stress (Marangoni flow [25]) and a streaming flow set up by the bubble oscillations [22], both of which are independent on buoyancy. Preliminary experiments with earth gravity $g_{e}$ show that oscillate boiling retain normal performance on a substrate inclining 30 degree [22] and even 90 degree (Fig. 1b) to

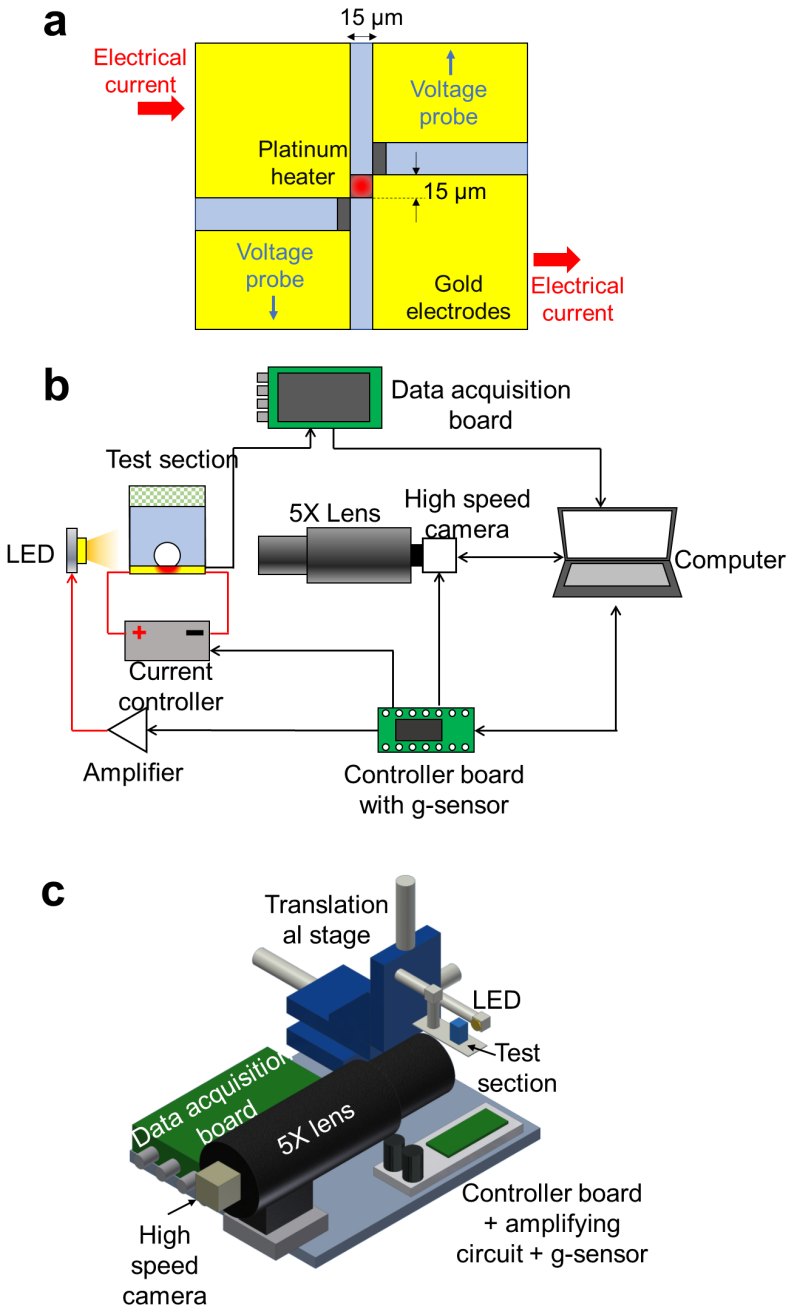

Fig. 2 (a) The design of the electrical microheater (b) Sketch of the experimental setup where red lines represent power connections and black lines represent flow of electrical signals. (c) 3D-view of the devices used.

horizontal direction. However, there has not been any study on the behavior of oscillate boiling in actual nonconventional gravity environment, i.e. low- $g$ and high- $g$.

In this work, we report on experimental observation of oscillate boiling in water both in low- $g$ and high- $g$ on-board parabolic flights. We then compare its behavior with results collected in terrestrial condition to draw conclusion on the effect of gravity on oscillate boiling. The experiments utilize a compact, simple, yet effective setup that can inspire future high-speed imaging experiments in low-gravity conditions. 


\section{Experimental apparatus}

\subsection{Experimental setup}

Figure 2 describes the complete experimental setup. The test section base was a glass wafer that contained a microfabricated $15 \times 15 \mu \mathrm{m}^{2}$ electrical heater made of platinum with gold electrodes wiring (see Fig. 2a). The heater's design and fabrication steps were the same as described in Ref [23]. Microscope cover glasses formed the side walls of the $1 \times 1 \times 1 \mathrm{~cm}^{3}$ liquid container. The container was filled with de-ionized water with bulk temperature of $\approx 17^{\circ} \mathrm{C}$. The top of the container was covered with a layer of foam to prevent any leakage of the liquid while equilibrating with the cabin pressure ( $\approx 0.8$ bar $)$. The electrical microheater was powered with a laser diode controller (Thorlabs LDC220) operating at constant current mode. The heater's instantaneous temperature was obtained from its resistance, which was recorded at a sampling rate of $10 \mathrm{MHz}$ with 12 bits accuracy with a data aquisition (DAQ) board (model USB 2020 from MCDAQ). The periodic drop of the temperature during each cycle (see below) allowed determining the bubble's oscillation frequency from the resistance signal.

The bubble oscillations were imaged with a sensitive CMOS camera (global shutter, model 1920-155um from Basler) equipped with a 5x-magnification lens (Makro lens MO-E65mm from Canon). This configuration provided a maximum temporal resolution of $1 \mathrm{~ms}$ and a spatial resolution of $1 \mu \mathrm{m}$. A typical period of the bubble oscillation is $4 \mu \mathrm{s}$, thus it demanded a short exposure time to capture the bubble dynamics free of motion blur. Because the minimum shutter duration of the camera was $100 \mu \mathrm{s}$, a strobed light source was synchronized with the camera. In particular, the camera exposure-out signal triggered a current amplifier [26] that generated a $1 \mu \mathrm{s}$-long pulse for the illuminating LED (Cree CXB1304). The $1 k H z$ triggering signal for the camera, the current controller, and the DAQ device were controlled with a microcontroller (Teensy 3.6). It was configured with a laptop through a USB port that also stored the images from the camera and waveforms from the DAQ board. An accelerometer (Adafruit LIS3DH) connected to the laptop measured the effective gravity and provided the signal to start the experiment.

Figure $2 \mathrm{~b}$ shows the electrical diagram and Fig. 2c shows the full mechanical design of the setup. The use of a data acquisition board and a USB-connected camera instead of an oscilloscope and a high speed camera saved weight, size and cost of the setup. The experiments enclosing dimension reduced to only $50 \times 30 \times$

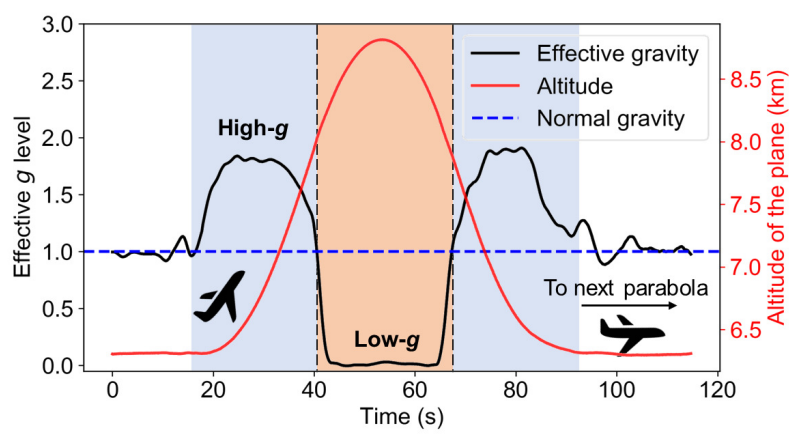

Fig. 3 (a) A typical profile of the aircraft altitude and effective gravity inside the cabin during one parabolic maneuver.

$25 \mathrm{~cm}^{3}$ with a total weight of $5 \mathrm{~kg}$. The setup may be used individually or, as we operated it, attached to a separate enclosed experiment. Its components can be easily assembled/dissembled during transportation

\subsection{Experimental procedure}

The experiments were conducted in November 2017 during the European Space Agency (ESA) $67^{\text {th }}$ parabolic flight campaign totalling 3 flights on board the Airbus A310 Zero-G. Each flight contained 31 parabolic maneuvers, each starting with an initial high- $g\left(1.8 g_{e}\right)$ pull-up, followed by a low- $g\left(0.01 g_{e}\right)$ period of 22 seconds, and ending with a high- $g\left(1.8 g_{e}\right)$ pullout. Data were obtained during normal- $g$, high- $g$, and low- $g$ phases (Fig. 3). In particular, once the gravity stabilized at a certain preset level for over 3 seconds, the microcontroller initialized simultaneously the camera, LED, current controller, and data acquisition board. The electrical and imaging data were stored locally inside the acquisition board and the camera. After a run time of $0.5 \mathrm{~s}$ the experiment ended and the data were transmitted to the computer. All equipment were then returned to the stand-by state waiting for the next run. Besides gravity, the electrical heating power was varied between $0 \mathrm{~mW}$ and $120 \mathrm{~mW}$ to probe the parameter space.

\section{Results and discussion}

3.1 Captured dynamics of oscillate boiling bubbles

Figures $4 \mathrm{a}$ and $4 \mathrm{~d}$ presents the dynamics of an oscillate boiling bubble at low- $g$ and high- $g$ driven at similar electrical powers of $63.8 \mathrm{~mW}$ and $64.1 \mathrm{~mW}$, repectively. The resulting maximum radius at low- $g$ is 13.2 $\mu \mathrm{m}$ and at high- $g$ is $13.6 \mu \mathrm{m}$. The bubble's dynamics are the same as in terrestrial condition (see Fig. 1a). During their oscillations, both bubbles release smaller 


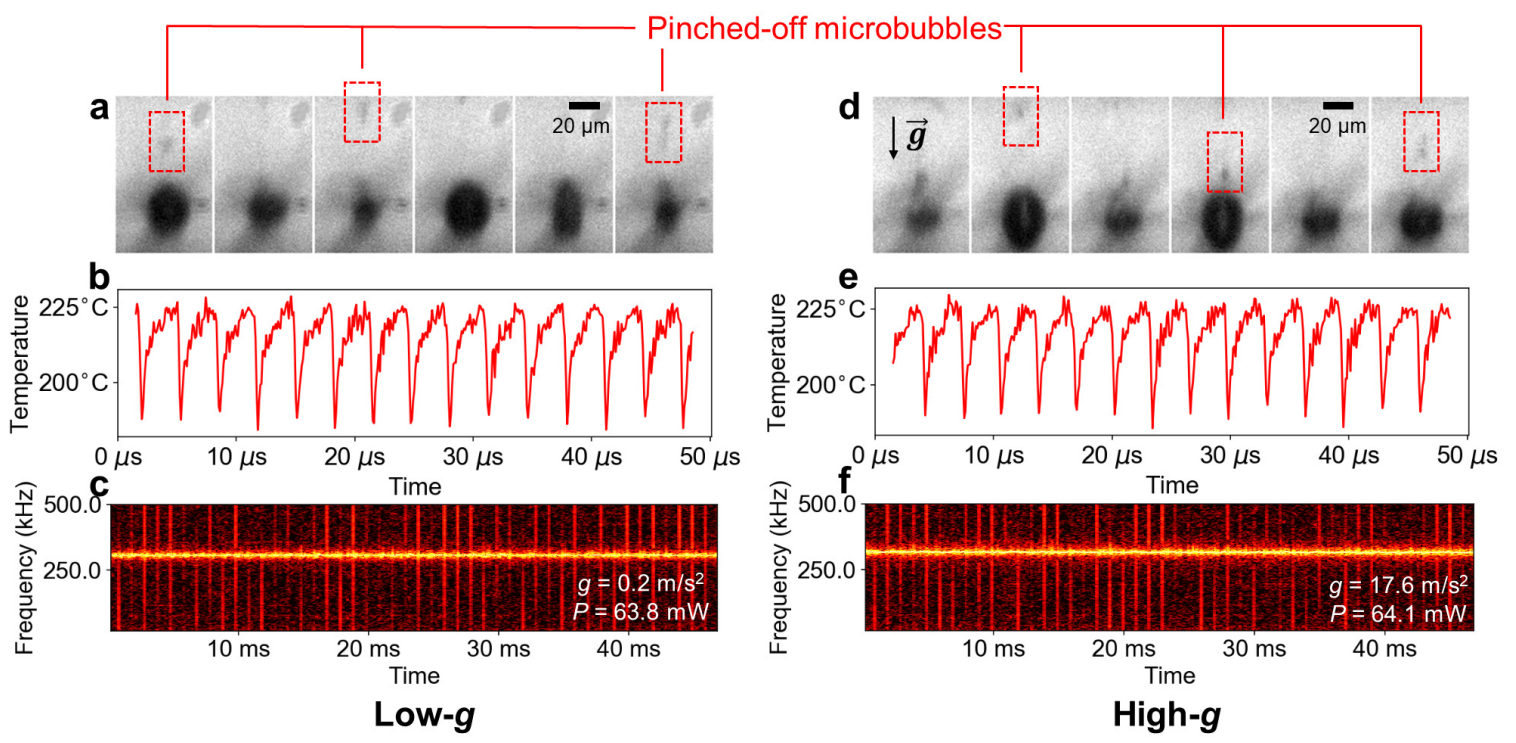

Fig. 4 Captured bubble dynamics of oscillate boiling bubbles, the temperature of the heating substrate and its frequency spectrogram in: (a-c) low- $g$ condition and (d-f) high- $g$ condition. Images from subfigure a and $d$ are taken from Video 1 and Video 2. The scale bar in subfigure a,d represents $20 \mu \mathrm{m}$, the time difference between two consecutive frames is $1 \mathrm{~ms}$ and the arrow indicate the orientation of the effective gravity inside the cabin. The high frequency noises that appears every $1 \mathrm{~ms}$ in subfigure $\mathrm{c}$ and $\mathrm{f}$ is induced by the current driving the LED at $1 \mathrm{kHz}$.

microbubbles at their apex, which are transported in a direction perpendicular to the substrate independent on the strength of gravity. Their oscillation is controlled only by the power supply and both stop once the electrical current is switched off after $0.5 \mathrm{~s}$. The recorded microheater's temperature is presented in Fig. 4b and 4e. When the bubble collapses, a liquid jet impacts onto the substrate and rapidly cools the heater: within less than $0.5 \mu \mathrm{s}$, the heater's temperature drops from $\approx 225^{\circ} \mathrm{C}$ to $\approx 185^{\circ} \mathrm{C}$, which is a cooling rate of $\approx 10^{8} \mathrm{~K} / \mathrm{s}$. Having received thermal energy from the vaporization of the liquid, the bubble re-expands and creates a dry spot below on the heater. The heater thus recovers to its peak temperature and the cycle repeats. A Fourier analysis of the temperature signal reveals the oscillation frequencies of the bubbles in the two gravity conditions, namely $305 \mathrm{kHz}$ and $310 \mathrm{kHz}$ (Fig. 4c and 4f).

\subsection{Parameters study}

By changing the electrical power, three regimes of oscillate boiling can be achieved. Below a threshold power the heater is not able to nucleate a bubble. Above $40 \mathrm{~mW}$ a bubble starts forming at the heating spot and undergoes unstable oscillation. In this unstable mode, the bubbles show neither microbubble pinch-off nor periodic cooling of the heater. After $\approx 10 \mathrm{~ms}$, the oscillations halt and the bubble grows similar to a conventional nucleate boiling bubble [23]. Once the input

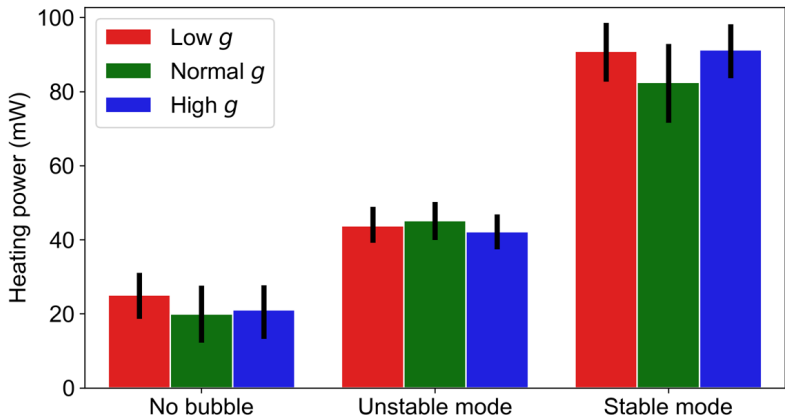

Fig. 5 Three oscillate boiling regimes at different input power and gravity conditions. The height of the column represents the mean power and the error bar represents the standard deviation of the sample. Each columns contained at least 8 data points.

power surpasses $60 \mathrm{~mW}$, stable oscillation featuring microbubble pinch off and microbubble streaming together with the periodic cooling of the heater is observed, see Fig. 4. Figure 5 categorizes the observed oscillate boiling regimes and plots their electrical current as a function of gravity. Overall we find very similar behavior with no clear dependency on gravity.

In the stable oscillate boiling mode the oscillation frequency decreases monotonically with increasing maximum bubble radius, which is also predicted with a simple model. The model is the same as proposed in Ref. [22], in which two main equations are used. The 


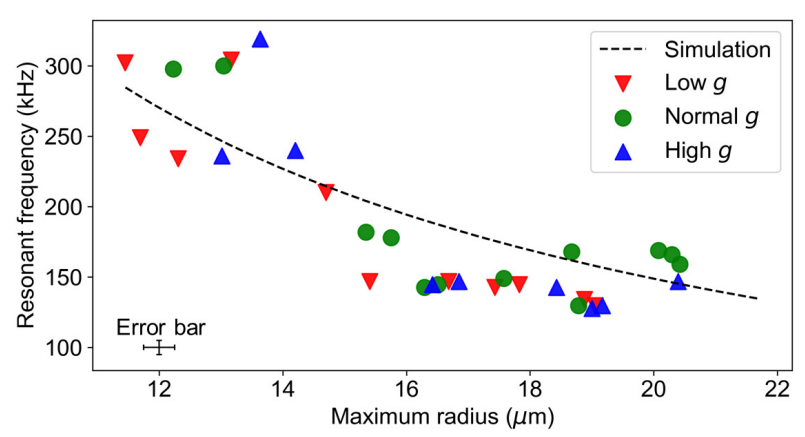

Fig. 6 The frequency-radius characteristics of oscillate boiling bubble in different gravity conditions. The simulation data follows the calculation reported in reference [22] with a change of ambient pressure to 0.8 bar, matching the cabin's pressure. All data points share the same experimental error and is indicated by the error bar plotted in the bottom-left corner.

first equation describes the evolution of the bubble's radius $R$, which follows Keller-Miksis model:

$$
\left(1-\frac{\dot{R}}{c}\right) R \ddot{R}+\frac{3}{2} \dot{R}^{2}\left(1-\frac{\dot{R}}{3 c}\right)=\left(1+\frac{\dot{R}}{c}\right) \frac{p_{l}}{\rho}+\frac{R}{\rho c} \frac{d p_{l}}{d t}(1)
$$

where $c$ is the sound velocity, $p_{l}$ is the liquid pressure and $\rho$ is the density of the bubble's inner gas-vapour mixture. The second equation describes energy conservation of the bubble's interior:

$p d V+d U=d Q$

where $p$ is the work done to the gas as the bubble expands/collapses, $V$ is the bubble volume, $U$ is the potential energy accounting for evaporation and condensation, $Q$ is the supplied energy from the electrical heater minus the heat transfer into liquid by diffusion and latent heat. At every time-step, these two equations are coupled to solved for two unknowns: namely the bubble's radius $R$ and inner temperature T. As presented in Ref. [22], each input power results in a stable's maximum radius and resonant frequency. Figure 6 presents the radius-frequency relationship when the input power varies, both experimentally for different gravity conditions and numerically from the model prediction. While there is some scatter, we find overall quantitative agreement with the model (black line in Fig. 6) and its independence of gravity.

\subsection{Discussion}

The analysis of the bubble's dynamics and its oscillation frequency shown in Figs. 5 and 6 demonstrates that its quantitative and qualitative characteristics are independent of the effective gravity conditions: high- $g$, low- $g$, and normal $g$. These bubble's behaviors might indicate the independence of the oscillate boiling regime on gravity within the ranges tested. We attribute this characteristics to the governing physics: the oscillation is driven by the bubble's inertia and the liquid jet impact, the rise of the microbubble stream is driven by the Marangoni flow along the bubble's interface and likely by a streaming flow caused by the bubble oscillation. These driving factors are independent of buoyancy. The experimental verification not only demonstrates the independence on gravity but also that the previously proposed explanations and model are sound [22].

Within our system, the working capacity of oscillate boiling is limited by the heater. Above a certain input power, the heater breaks, likely because of thermal stress and mechanical stress induced by the bubble's collapse. The maximum power achieved in this experiment is $120 \mathrm{~mW}$ with a $15 \times 15 \mu \mathrm{m}^{2}$ heater size, corresponding to a heat flux of $10^{8} \mathrm{~W} / \mathrm{m}^{2}$. This number is significantly higher as compared to previously reported critical heat fluxes of $10^{5} \mathrm{~W} / \mathrm{m}^{2}$ for water [4], $10^{6} \mathrm{~W} / \mathrm{m}^{2}$ for FC-72 [6], and $\mathrm{nPFH}[27,15]$. This may be partly explained that oscillate boiling operates above the critical Leidenfrost temperature [23].

One limiting factor of oscillate boiling is that the size of the heat source must be smaller than $20 \mu \mathrm{m}$ $[22,23]$. Currently only miniature devices such as high power transistors or microfluidic platforms may benefit from this boiling mode. The next challenge is to scaleup the regime, thus to widen the range of application.

\section{Conclusion}

Experiments in various gravity conditions on-board parabodic flights confirm the independence of the oscillate boiling $\quad{ }_{279}$ bubble on gravity. In low- $g$ and high- $g$ conditions, os- 280 cillate boiling bubble retains its characteristic behavior $\quad{ }_{281}$ and operation limits. The gravity-free feature together ${ }_{282}$ with its high thermal efficiency encourage the devel- ${ }^{283}$ opment of oscillate-boiling-based heat transfer devices ${ }_{284}$ for applications in terrestrial and outer space environ- 285 ments. To achieve this, the driving, packing, and the ${ }_{286}$ interaction of many heaters and oscillate boiling bub- $\quad 287$ bles should be be studied.

Acknowledgements We thank ESA for organizing the 67th parabola flight campaign and Danail Obreschkow for his great help and company during the campaign. The project received financial support from the Ministry of Education, Singapore (Tier 1160 grant RG90/15), the School of Physical and Mathematical Sciences, Nanyang Technological University and the Swiss National Science Foundation (grant no. 513234). 


\section{References}

1. Issam Mudawar. Two-phase microchannel heat sinks: theory, applications, and limitations. Journal of electronic packaging, 133(4):041002, 2011.

2. Jungho Kim. Review of nucleate pool boiling bubble heat transfer mechanisms. International Journal of Multiphase Flow, 35(12):1067-1076, 2009.

3. Yoshiyuki Abe and Akira Iwasaki. Pool boiling under microgravity. Advances In Space Research, 13(7):165168, 1993.

4. H Ohta, K Kawasaki, S Okada, H Azuma, S Yoda, and $\mathrm{T}$ Nakamura. On the heat transfer mechanisms in microgravity nucleate boiling. Advances in Space Research, 24(10):1325-1330, 1999.

5. T Oka, Y Abe, YH Mori, and A Nagashima. Pool boiling of n-pentane, cfc-113, and water under reduced gravity: parabolic flight experiments with a transparent heater. Journal of heat transfer, 117(2):408-417, 1995.

6. Jungho Kim, John F Benton, and Derek Wisniewski. Pool boiling heat transfer on small heaters: effect of gravity and subcooling. International Journal of Heat and Mass Transfer, 45(19):3919-3932, 2002.

7. Christopher D Henry and Jungho Kim. A study of the effects of heater size, subcooling, and gravity level on pool boiling heat transfer. International journal of heat and fluid flow, 25(2):262-273, 2004.

8. CD Henry, J Kim, and J McQuillen. Dissolved gas effects on thermocapillary convection during boiling in reduced gravity environments. Heat and mass transfer, 42(10):919-928, 2006.

9. JF Zhao, Gang Liu, SX Wan, and N Yan. Bubble dynamics in nucleate pool boiling on thin wires in microgravity. Microgravity Science and Technology, 20(2):81-89, 2008.

10. Rishi Raj and Jungho Kim. Heater size and gravity based pool boiling regime map: transition criteria between buoyancy and surface tension dominated boiling. Journal of Heat Transfer, 132(9):091503, 2010.

11. RR Souza, Júlio César Passos, and EM Cardoso. Confined and unconfined nucleate boiling under terrestrial and microgravity conditions. Applied Thermal Engineering, 51(1-2):1290-1296, 2013.

12. Xiande Fang, Ling Zheng, Yan He, Gen Li, Minghua Bi, Bei Yang, and Xi Wang. Experimental study of pool boiling critical heat flux on thin wires under various gravities. Microgravity Science and Technology, pages 1-7, 2019.

13. Olivier Kannengieser, Catherine Colin, and Wladimir Bergez. Pool boiling with non-condensable gas in microgravity: results of a sounding rocket experiment. $M i-$ crogravity Science and Technology, 22(3):447-454, 2010.

14. Yan-Fang Xue, Jian-Fu Zhao, Jin-Jia Wei, Jing Li, Dong Guo, and Shi-Xin Wan. Experimental study of nucleate pool boiling of fc-72 on smooth surface under microgravity. Microgravity Science and Technology, 23(1):75, 2011.

15. Vijay Kumar Dhir, Gopinath R Warrier, Eduardo Aktinol, David Chao, Jeffery Eggers, William Sheredy, and Wendell Booth. Nucleate pool boiling experiments (npbx) on the international space station. Microgravity Science and Technology, 24(5):307-325, 2012.

16. Rishi Raj, Jungho Kim, and John McQuillen. Pool boiling heat transfer on the international space station: Experimental results and model verification. Journal of Heat Transfer, 134(10):101504, 2012.

17. Christopher Konishi and Issam Mudawar. Review of flow boiling and critical heat flux in microgravity. International Journal of Heat and Mass Transfer, 80:469-493, 2015 .
18. Catherine Colin, Olivier Kannengieser, Wladimir Bergez, Michel Lebon, Julien Sebilleau, Michaël Sagan, and Sébastien Tanguy. Nucleate pool boiling in microgravity: Recent progress and future prospects. Comptes Rendus Mécanique, 345(1):21-34, 2017.

19. M Steinbichler, S Micko, and J Straub. Nucleate boiling heat transfer on a small hemispherical heater and a wire under microgravity. In Heat Transfer Conference, volume 2, pages 539-544, 1998.

20. Ho Sung Lee, Herman Merte, and Francis P Chiaramonte. Pool boiling phenomena in microgravity. In Heat Transfer Conference, volume 2, pages 395-400, 1998.

21. Johannes Straub. Origin and effect of thermocapillary convection in subcooled boiling. Annals of the New York Academy of Sciences, 974(1):348-363, 2002.

22. Fenfang Li, S Roberto Gonzalez-Avila, Dang Minh Nguyen, and Claus-Dieter Ohl. Oscillate boiling from microheaters. Physical Review Fluids, 2(1):014007, 2017.

23. Dang Minh Nguyen, Liangxing $\mathrm{Hu}$, Jianmin Miao, and Claus-Dieter Ohl. Oscillate boiling from electrical microheaters. Physical Review Applied, 10(4):044064, 2018.

24. John R Blake and DC Gibson. Cavitation bubbles near boundaries. Annual review of fluid mechanics, 19(1):99$123,1987$.

25. LE Scriven and CV Sternling. The marangoni effects. Nature, 187(4733):186, 1960

26. Christian Willert, Boleslaw Stasicki, Joachim Klinner, and S Moessner. Pulsed operation of high-power light emitting diodes for imaging flow velocimetry. Measurement Science and Technology, 21(7):075402, 2010.

27. Rishi Raj, Jungho Kim, and John McQuillen. Subcooled pool boiling in variable gravity environments. Journal of Heat Transfer, 131(9):091502, 2009. 